\title{
Multi-wavelength campaign on NGC 7469
}

\section{The rich $640 \mathrm{ks}$ RGS spectrum}

\author{
Ehud Behar ${ }^{1,2}$, Uria Peretz ${ }^{1}$, Gerard A. Kriss ${ }^{3}$, Jelle Kaastra ${ }^{4,5}$, Nahum Arav ${ }^{6,1}$, Stefano Bianchi ${ }^{7}$, \\ Graziella Branduardi-Raymont ${ }^{8}$, Massimo Cappi ${ }^{9}$, Elisa Costantini ${ }^{4}$, Barbara De Marco ${ }^{10}$, Laura Di Gesu ${ }^{11}$, \\ Jacobo Ebrero $^{12}$, Shai Kaspi ${ }^{13}$, Missagh Mehdipour ${ }^{4}$, Stéphane Paltani ${ }^{11}$, Pierre-Olivier Petrucci ${ }^{14}$, \\ Gabriele Ponti ${ }^{10}$, and Francesco Ursini ${ }^{14}$ \\ 1 Department of Physics, Technion, 32000 Haifa, Israel \\ e-mail: behar@physics.technion.ac.il \\ 2 Department of Astronomy, University of Maryland, College Park, MD 20742-2421, USA \\ 3 Space Telescope Science Institute, 3700 San Martin Drive, Baltimore, MD 21218, USA \\ ${ }^{4}$ SRON Netherlands Institute for Space Research, Sorbonnelaan 2, 3584 CA Utrecht, The Netherlands \\ 5 Leiden Observatory, Leiden University, PO Box 9513, 2300 RA Leiden, The Netherlands \\ 6 Department of Physics, Virginia Tech, Blacksburg, VA 24061, USA \\ 7 Dipartimento di Matematica e Fisica, Università degli Studi Roma Tre, via della Vasca Navale 84, 00146 Roma, Italy \\ 8 Mullard Space Science Laboratory, University College London, Holmbury St. Mary, Dorking, Surrey, RH5 6NT, UK \\ 9 INAF-IASF Bologna, via Gobetti 101, 40129 Bologna, Italy \\ ${ }^{10}$ Max-Planck-Institut für extraterrestrische Physik, Giessenbachstrasse, 85748 Garching, Germany \\ 11 Department of Astronomy, University of Geneva, 16 chemin d'Ecogia, 1290 Versoix, Switzerland \\ 12 European Space Astronomy Centre, PO Box 78, 28691 Villanueva de la Cañada, Madrid, Spain \\ 13 School of Physics and Astronomy, Tel Aviv University, 69978 Tel Aviv, Israel \\ ${ }^{14}$ Univ. Grenoble Alpes, CNRS, IPAG, 38000 Grenoble, France
}

Received 22 October 2016 / Accepted 22 December 2016

\begin{abstract}
Aims. Outflows in active galaxies (AGNs) are common, although their launching mechanism, location, and physical impact on the host galaxy remain controversial. We conducted a multi-wavelength six-month campaign to observe the nearby Seyfert galaxy NGC 7469 with several observatories in order to better understand and quantify the outflow in this AGN.

Methods. We report on the time-integrated line-resolved X-ray spectrum of NGC 7469 obtained with the Reflection Grating Spectrometer (RGS) on board XMM-Newton. We used the RGS spectrum to discern the many AGN outflow components and applied a global fit to obtain their physical parameters.

Results. We find that the AGN wind can be well described by three narrow velocity components at $\sim-650,-950$, and $-2050 \mathrm{~km} \mathrm{~s}^{-1}$. The RGS clearly resolves the $-2050 \mathrm{~km} \mathrm{~s}^{-1}$ component in $\mathrm{C}^{5+} \mathrm{Ly} \alpha$, while the $-650 \mathrm{~km} \mathrm{~s}^{-1}$ and $-950 \mathrm{~km} \mathrm{~s}^{-1}$ velocities are blended. Similar velocities $\left( \pm 200 \mathrm{~km} \mathrm{~s}^{-1}\right)$ are resolved in the UV. The H-equivalent column densities of these components are, respectively, $N_{\mathrm{H}} \sim 7 \times 10^{20}, 2.2 \times 10^{21}$, and $10^{20} \mathrm{~cm}^{-2}$, for a total of $\sim 3 \times 10^{21} \mathrm{~cm}^{-2}$, which was also measured in 2004 , indicating the absorber did not significantly change. The $-650 \mathrm{~km} \mathrm{~s}^{-1}$ component shows a broad ionization distribution $(-1 \lesssim \log \xi \lesssim 2, \xi$ being the ionization parameter in $\left.\mathrm{erg} \mathrm{s}^{-1} \mathrm{~cm}\right)$. We identify a photo-ionized emission component blue-shifted by $\sim-450 \mathrm{~km} \mathrm{~s}^{-1}$, somewhat broad $(F W H M=$ $1400 \mathrm{~km} \mathrm{~s}^{-1}$ ), and with $-1 \lesssim \log \xi \lesssim 1 \mathrm{erg} \mathrm{s}^{-1} \mathrm{~cm}$, which we ascribe to the same outflow that produces the absorption lines. We also find a collisionally ionized component at $k T=0.35 \mathrm{keV}$ that we associate with the circum-nuclear star-formation activity of NGC 7469, as it follows the $L_{\mathrm{FIR}} / L_{\mathrm{X}} \approx 10^{4}$ relation found in star forming galaxies. The elemental abundance ratios of C, N, Ne, S, and $\mathrm{Fe}$ to $\mathrm{O}$ in the outflow tend to be between one and two times solar. Preliminary estimates of the absorber distance from the AGN center suggest it is at least a few pc away from the center, but more advanced methods need to be applied in order to obtain better constraints.

Conclusions. The complex X-ray spectrum of NGC 7469 demonstrates the richness of high energy phenomena taking place in AGN cores. The subtle spectroscopic differences between the various components require deep, high-resolution observations, such as the present RGS spectrum, if one is to resolve them and perform quantitative plasma diagnostics.
\end{abstract}

Key words. galaxies: active - quasars: absorption lines - galaxies: Seyfert - galaxies: individual: NGC 7469

\section{Introduction}

AGN outflows couple supermassive black holes to their environments (e.g., Di Matteo et al. 2005). Despite many years of spectroscopic observations in the X-rays and in the UV, the emerging picture of these outflows is still ambiguous. Spectra are typically characterized by several kinematic and ionization components that are readily measured with contemporary instruments (see Crenshaw et al. 2003, for a review). However, the relation between the various components remains debatable. Reaching a more complete physical picture of active galactic nucleus (AGN) outflows has proven to be challenging (e.g., Blustin et al. 2005). 
Thus, their impact, perhaps most interestingly their mass outflow rate that affects their momentum and energy feedback potential to the host galaxy remains largely unknown.

Seyfert outflows, for the most part, eject winds of a few hundred $\mathrm{km} \mathrm{s}^{-1}$, and with $v / c<0.01$ where $c$ is the speed of light (Kaastra et al. 2000; Kaspi et al. 2001). Their absorption spectra are extremely rich and can feature essentially all charge states from neutral to H-like ions. These winds thus appear to be different from the ultra high velocity outflows $(v / c \sim 0.1)$ observed mostly through their $\mathrm{Fe}^{25+}$ absorption (Reeves et al. 2003; Tombesi et al. 2010). Since the mechanical power in AGN outflows scales with the mass outflow rate and with $v^{2}$, and since the luminosity $L$ of an AGN scales with the mass accretion rate and $c^{2}$, mass outflow rates in Seyferts need to be 10000 times higher than the accretion rate for their mechanical power to reach the AGN luminosity. On the other hand, the high-resolution high-signal-to-noise ratio $(\mathrm{S} / \mathrm{N})$ spectra observed in Seyferts serve as fertile grounds for studying the physics of outflows from black-hole accretion sources in the greatest detail possible.

The theoretical models of AGN outflows vary greatly. The launching mechanism remains controversial, as thermal evaporation of the torus (e.g., Krolik \& Kriss 2001), radiation pressure (e.g., Proga et al. 2000), and magnetohydrodynamics (MHD) driving (e.g., Contopoulos \& Lovelace 1994) have all been proposed. Nevertheless, matching observed spectra with a real physical model has proven to be difficult (e.g., Sim et al. 2008). Very different physical models have been successful in producing the broad ionization distribution, both with MHD winds (Fukumura et al. 2010), and with radiation compression (Różańska et al. 2006; Stern et al. 2014). The kinematic structure obtained when fitting for discrete velocity and ionization components in each AGN remains harder to explain from first principles, as those do not seem to follow any obvious trend (Blustin et al. 2005; McKernan et al. 2007). Laha et al. (2014) find interesting trends between targets of outflow velocity and column density that increase with ionization parameter, which could represent an important clue to identifying the physical mechanism of these winds.

The mass outflow rate depends on the location (and density) of the ionized absorber. Since X-ray imaging does not resolve line-of-sight absorbers, several methods have been proposed and used to estimate the distance from the center $(r)$ or the $\mathrm{H}$ density $\left(n_{\mathrm{H}}\right)$ and thus to lift the hampering degeneracy between the two, which is imposed by the measured ionization parameter $\xi=L /\left(n_{\mathrm{H}} r^{2}\right)$. The most accurate of these methods is identification of absorption from density-sensitive metastable levels (Arav et al. 2001, 2015). However, in the X-rays, most such levels require high densities that are not observed, and low-density diagnostics have proven to be extremely complicated to implement (Kaastra et al. 2004). Associating the absorber with the narrow emission lines is yet another method. Fluxes of lines driven by recombination scale with $n_{\mathrm{H}}^{2}$, while the column density scales with $n_{\mathrm{H}}$, which allows one to obtain the density (e.g., Behar et al. 2003). However, this method works only when there are bright emission lines. Another common approach is to estimate recombination or ionization times as the absorber responds to changes in the ionizing flux. In many cases, the ionization of the absorber does not change on short time scales, despite variability of the continuum (Behar et al. 2003; Netzer et al. 2003; Ebrero et al. 2010; Kaastra et al. 2012), which constrains it to be many pc away from the nucleus, and the density to be low. Other works do suggest a response of the absorber ionization to the continuum level that places the absorber closer than $6 \mathrm{pc}$ in NGC 3783 (Krongold et al. 2005), and as close as a few light days in NGC 4051 (Krongold et al. 2007). For the most part, non-continuous monitoring impedes transient ionization/recombination modeling, and one needs to revert to statistical correlations between flux level and ionization state (Krongold et al. 2007), or column density of certain ions (Kaastra et al. 2012).

The present campaign aims to harness XMM-Newton, HST, Chandra, NuSTAR, and Swift, as well as ground based telescopes, to monitor the changes of the continuum and the response of the ionized absorber of NGC 7469. Similar successful campaigns in the past include other Seyfert galaxies, such as NGC 3783 (Netzer et al. 2003), Mrk 279 (Gabel et al. 2005; Ebrero et al. 2010), Mrk 509 (Detmers et al. 2011; Kaastra et al. 2012), and NGC 5548 (Kaastra et al. 2014).

The unique properties of NGC 7469 make it the ideal candidate to respond on shorter, better-monitored time scales. NGC 7469 is as luminous as $\operatorname{Mrk} 509\left(L_{\text {bol }} \approx 10^{45} \mathrm{erg} \mathrm{s}^{-1}\right)$, but hosts a black hole that is ten times smaller $\left(M_{\mathrm{BH}}=10^{7} M_{\odot}\right)$, resulting in a high Eddington ratio of $L / L_{\text {Edd }} \approx 0.3$. Given this compactness, one expects ten times faster variability. Indeed, the normalised excess variance of NGC 7469 is ten times higher than that of Mrk 509 on short time scales of $<100 \mathrm{ks}$ (Ponti et al. 2012). In the $0.3-10 \mathrm{keV}$ band, NGC 7469 is one of the ten brightest Seyfert 1 galaxies with a flux of 4-5 $\times$ $10^{-11} \mathrm{erg} \mathrm{s}^{-1} \mathrm{~cm}^{-2}$.

Previous analyses of X-ray Chandra and XMM-Newton grating spectra of NGC 7469 were published by Scott et al. (2005) and by Blustin et al. (2007), respectively. Blustin et al. (2007) found a wide range of ionization, with $-0.5<\log \xi<$ $3.5\left(\mathrm{erg} \mathrm{s}^{-1} \mathrm{~cm}\right)$, and two main velocity regimes, at $\sim-650$ and $-2300 \mathrm{~km} \mathrm{~s}^{-1}$, the former also having been identified by Scott et al. (2005). The total absorbing column density in the $\mathrm{X}$ rays was of order $3 \times 10^{21} \mathrm{~cm}^{-2}$. The previous works also identified the lowest ionization phase of the X-ray absorber with one of the phases of the UV absorber. They estimated the outflow to be at the base of the torus. In this paper, we revisit the ionized absorber of NGC 7469 and focus on the co-added $640 \mathrm{ks} \mathrm{spec-}$ trum, acquired with seven separate observations of the Reflection Grating Spectrometer (RGS) on board XMM-Newton. The time dependence, as well as the simultaneous UV and hard Xray spectra, will be discussed in future companion papers.

We take the rest frame of NGC 7469 to be at a redshift of $z=0.016268$, based on the $21 \mathrm{~cm}$ line (Springob et al. 2005), and the luminosity distance to be $65.1 \mathrm{Mpc}$. For the total Galactic column density towards NGC 7469, we add the H I column of $4.34 \times 10^{20} \mathrm{~cm}^{-2}$ (Wakker et al. 2011) and twice the measured $\mathrm{H}_{2}$ column density of $5.75 \times 10^{19} \mathrm{~cm}^{-2}$ (Wakker 2006), for a total $\mathrm{H}$ column of $5.5 \times 10^{20} \mathrm{~cm}^{-2}$. For the solar abundances, we refer to Asplund et al. (2009). The remainder of the paper includes the data reduction (Sect. 2), the fitting method (Sect. 3), the results (Sect. 4), and discussion (Sect. 5).

\section{Data and reduction}

XMM-Newton observed NGC 7469 seven times for approximately $90 \mathrm{ks}$ on average each time, and with varying spacings between observations, in order to probe variability on different time scales. Luckily, no major background flares occurred during these observations, which allowed us to essentially make use of the entire exposure time. The observation log is given in Table 1 , including the mean RGS1 source count rates of each observation. It can be seen that in terms of the RGS count rate, the source varied by up to $30 \%$ between observations. No obvious change 
Table 1. Observation log.

\begin{tabular}{cccc}
\hline \hline $\begin{array}{c}\text { Observation } \\
\text { id }\end{array}$ & Start date & $\begin{array}{c}\text { Duration } \\
(\mathrm{ks})\end{array}$ & $\begin{array}{c}\text { RGS1 count rate } \\
(\mathrm{cts} / \mathrm{s})\end{array}$ \\
\hline 0760350201 & 2015-Jun.-12 & 89.5 & 0.685 \\
0760350301 & 2015-Nov.-24 & 85.6 & 0.600 \\
0760350401 & 2015-Dec.-15 & 84.6 & 0.521 \\
0760350501 & 2015-Dec.-23 & 89.5 & 0.536 \\
0760350601 & 2015-Dec.-24 & 91.5 & 0.593 \\
0760350701 & 2015-Dec.-26 & 96.7 & 0.580 \\
0760350801 & 2015-Dec.-28 & 100.2 & 0.620 \\
\hline
\end{tabular}

in the absorption features was identified. If they did vary, these were subtle changes that will be investigated in a separate paper. This allowed us to co-add all RGS (both 1 and 2) spectra and to study the mean RGS features at a high S/N. We did verify that the global model fitted to the mean RGS spectrum, as discussed below, fits each individual spectrum well. In statistical terms, after merely normalizing the model continuum, the $\chi^{2} /$ d.o.f. remains between 1.30 and 1.57 for all individual spectra, while the best fit model to the combined spectrum yields $\chi^{2} /$ d.o.f. $=1.40$.

We reduced each RGS (1 and 2) spectrum with the rgsproc task of the standard XMM/SAS pipeline tool version 14 (xmmsas_20141104_1833). The default spectral binning presented in this work is of $20 \mathrm{~m} \AA$, which over-samples the instrumental line spread function by a factor of a few. All seven RGS spectra were subsequently combined using the rgscombine task. The present paper focuses on this combined, mean spectrum. Each RGS observation had a slightly different pointing. This was done on purpose to dither the chip gaps on the RGS detectors. Indeed, the combined fluxed spectrum has no striking gaps, with some residuals just barely noticed as narrow jumps in the spectrum at $9.9,13.1,20.75$, and $22.7 \AA$. The mean energy and photon flux measured with the combined (mean) RGS spectrum between 0.3 and $2.5 \mathrm{keV}$ is $3.15 \times 10^{-11} \mathrm{erg} \mathrm{s}^{-1} \mathrm{~cm}^{-2}$, and $0.027 \mathrm{ph} \mathrm{s}^{-1} \mathrm{~cm}^{-2}$, respectively.

\section{Fitting approach}

We conducted a global fit to the total RGS spectrum between 7 and $38 \AA$ using the $X$ spec $11^{1}$ software package. Both the $\mathrm{C}$ and $\chi^{2}$ statistic for fitting were tested. Given the high $\mathrm{S} / \mathrm{N}$, they yield essentially the same results. From here on, we refer only to the $\mathrm{C}$ statistic. We started with a Galactically absorbed powerlaw continuum. We also tested whether or not a soft-excess (e.g., black body) component improves the fit. Within the limited RGS band, it does not, but a broader perspective that includes hard X-rays and UV does require such a component (Mehdipour et al., in prep.) and will be modeled properly in a subsequent paper (Petrucci et al., in prep.).

We subsequently used the analytical version (2.11n8) of the XSTAR photo-ionized plasma models (Kallman \& Bautista 2001). These models employ pre-calculated photo-ionization balance results, using a template power-law spectrum with a photon index of $\Gamma=2$, so they do not fully reflect the actual broad-band spectrum of each source. Nonetheless, the present best-fit model, limited to the RGS band, has $\Gamma=2.17$ and no soft excess, so we expect this to be a good approximation. We find that six (warmabs) photo-ionized absorption components, and two (photemis) emission components provide a satisfactory

\footnotetext{
1 http://heasarc.nasa.gov/xanadu/xspec/
}

description of the spectrum. Since we identify ionized absorption at the observed frame, likely due to local hot gas, we also add a hotabs component at $z=0$. For more information about the models, we refer the reader to the XSTAR website ${ }^{2}$. Finally, residuals of Fe L-shell emission lines motivated the addition of a collisionally-ionized component.

Simultaneous UV spectra of NGC 7469 reveal three main outflow velocity components at $-540,-857$, and $-1865 \mathrm{~km} \mathrm{~s}^{-1}$ that are approximately 60,35 , and $55 \mathrm{~km} \mathrm{~s}^{-1}(\sigma)$ wide, and a fourth weak one at $-1400 \mathrm{~km} \mathrm{~s}^{-1}$. We used the three main UV velocity shifts and their broadening as a starting point for the corresponding parameters of the X-ray absorber model fit, and added ionization components at each velocity as required by the data. However, it is important to keep in mind that the spectral resolution of the RGS is approximately $\Delta \lambda=70 \mathrm{~m} \AA$ across the band. Consequently, its kinematic resolving power around $\lambda=20 \AA$ is approximately $v=(\Delta \lambda / \lambda) c \approx 1000 \mathrm{~km} \mathrm{~s}^{-1}$.

We aim to measure both the column density $N_{\mathrm{H}}$ of each absorption component and the abundances of those elements that feature strong absorption and emission lines. Since no discrete $\mathrm{H}$ features appear in the X-ray spectrum, our result for $N_{\mathrm{H}}$ scales with the overall metallicity. Thus, in the model, $N_{\mathrm{H}}$ can be degenerate with the metal abundances. We therefore froze the abundance of $\mathrm{O}$, that has many features in the spectrum, to its solar value, and fitted for $N_{\mathrm{H}}$ and for the other abundances. The abundances in all absorption and photo-ionized emission components were tied together, assuming they are all the same. The abundances of the local absorption components (neutral and ionized) were kept at their solar values.

\section{Results}

The data and best-fit model are plotted in Fig. 1. It can be seen that the overall fit is good, albeit with some remaining residuals around $\sim 23 \AA$, and long-ward of $30 \AA$. The best-fit model yields a C statistic of 2151 (or $\chi^{2}=2179$ ) with 1550 spectral bins and 1528 degrees of freedom. The best-fit parameters are presented and discussed in the following sections. In Fig. 2, we plot the ratio of the data to the Galactically absorbed continuum, in order to demonstrate the ionized absorption and emission. The relatively shallow absorption ( 20\%) and bright emission lines can easily be discerned.

In Figs. 3 and 4, we zoom into the different regions of the spectrum to highlight the identified features. Here too, the fit can be seen to capture most features accurately. A notable exception is around the neutral $\mathrm{O}$ edge at $\sim 23 \AA$, where the continuum seems to be too low. The residuals above $30 \AA$ can be attributed predominantly to two unidentified emission lines at $32.6 \AA$ and at $33.4 \AA$, which we discuss below in Sect. 4.7 in more detail. These shortcomings of the model should have a negligible effect, if any, on the properties of the absorber (and narrow line emitter) that are the main focus of the paper.

\subsection{Kinematics}

Using three components (as in the UV), we obtain best-fit velocities of $-650 \pm 50 \mathrm{~km} \mathrm{~s}^{-1},-950_{-100}^{+50} \mathrm{~km} \mathrm{~s}^{-1}$, and $-2050_{-160}^{+50} \mathrm{~km} \mathrm{~s}^{-1}$. These velocities are slightly higher $\left(100-200 \mathrm{~km} \mathrm{~s}^{-1}\right)$ than the UV values, but consistent within the measurement and wavelength calibration $(<10 \mathrm{m \AA})$ uncertainties. Forcing the velocities to the UV values worsens the fit by $\Delta C \approx 20$ for more than

\footnotetext{
2 https://heasarc.gsfc.nasa.gov/xstar/xstar.html
} 


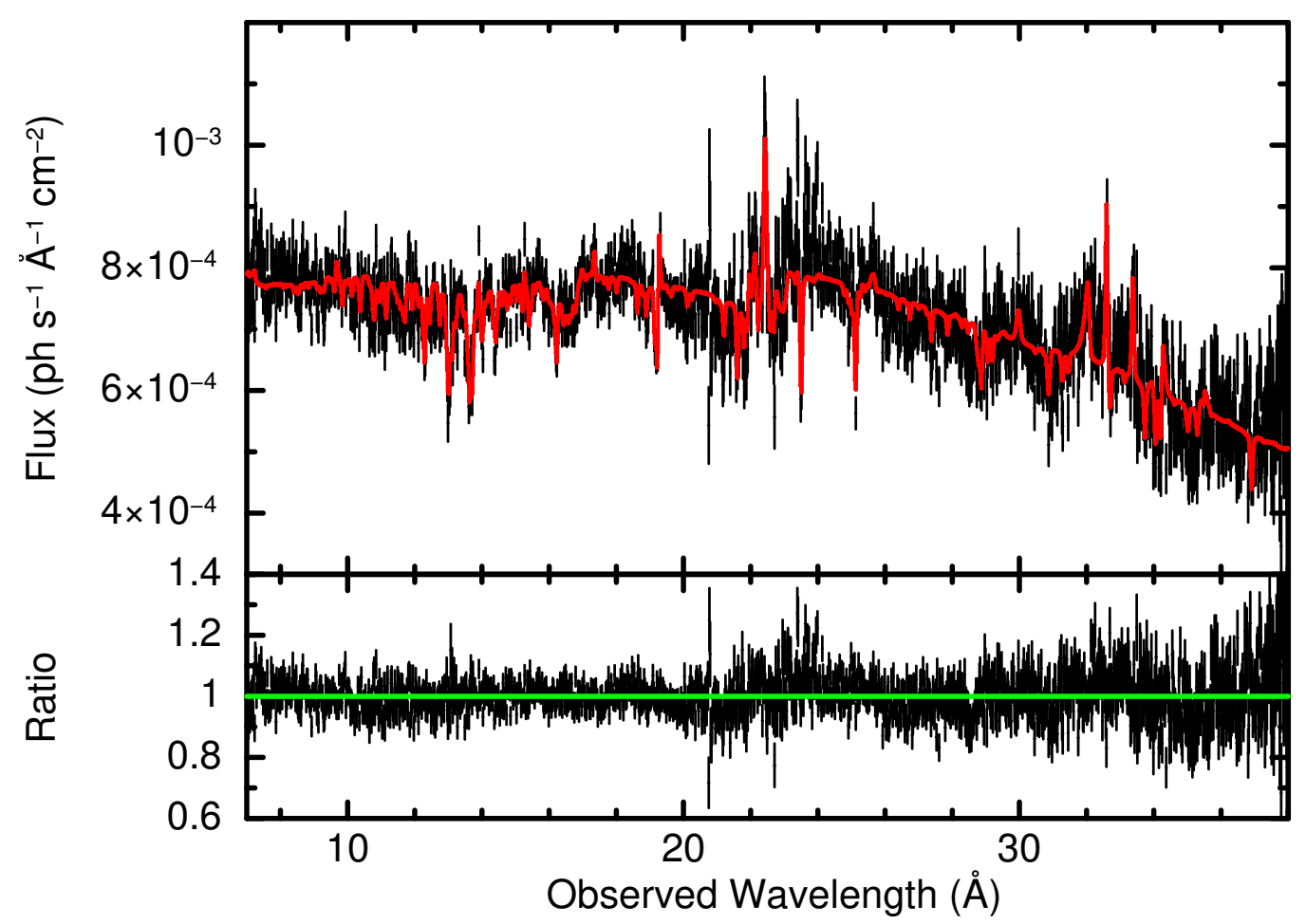

Fig. 1. Total RGS spectrum plotted with $20 \mathrm{~m} \AA$ bins, and the best-fit model overlaid, with the data/model ratio in the lower panel.

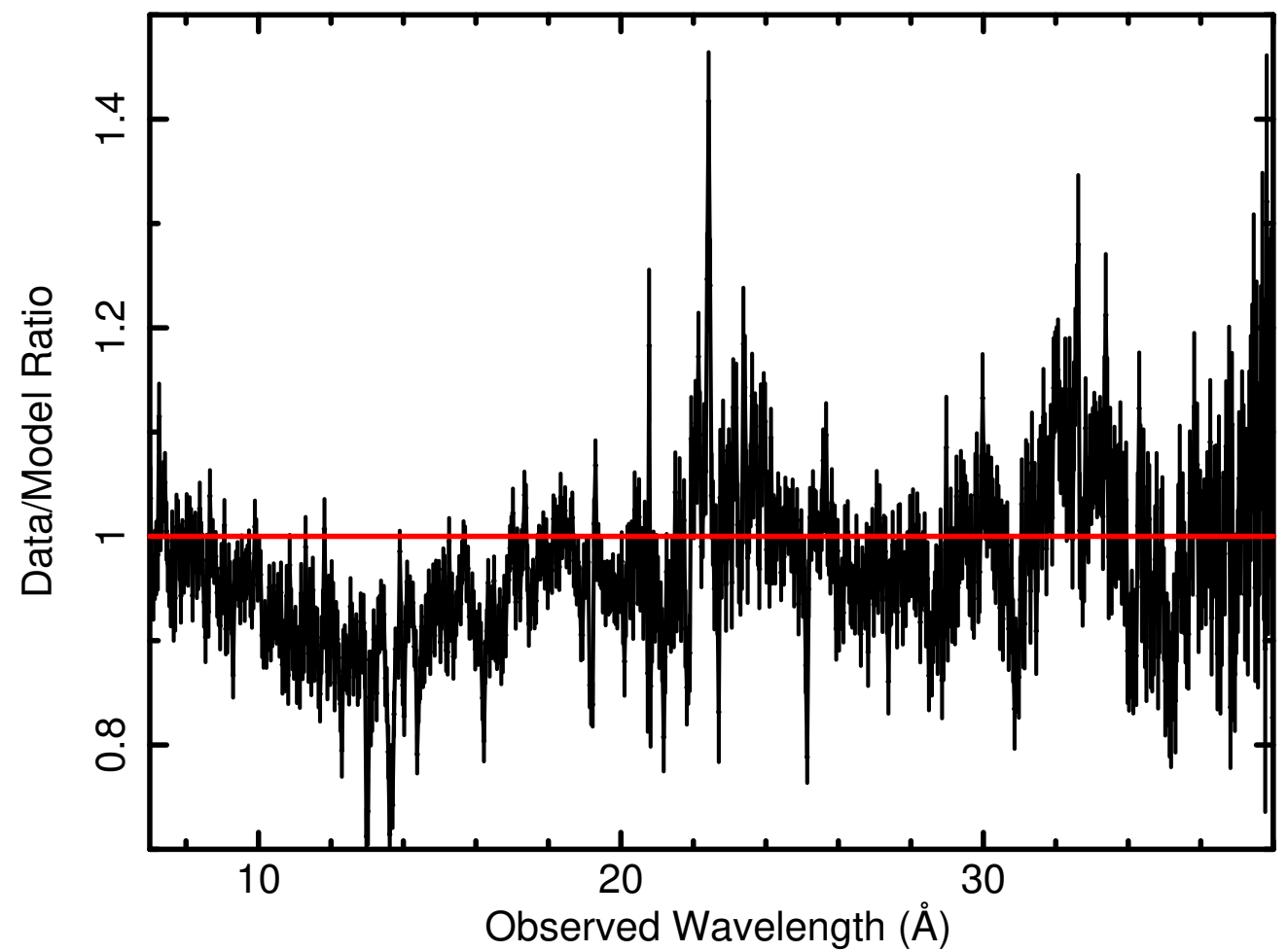

Fig. 2. Ratio of RGS spectrum to the Galactically absorbed continuum model plotted with $40 \mathrm{~m} \AA$ bins, demonstrating the effect of ionized absorption and emission.

1500 degrees of freedom. They are also in line with previous X-ray measurements (Scott et al. 2005; Blustin et al. 2007), suggesting that they have not varied for over more than a decade. Perhaps the best absorption (and emission) lines in the present spectrum for kinematic studies are the Ly $\alpha$ lines (close doublets, in fact) of $\mathrm{H}$-like $\mathrm{O}^{7+}$ and $\mathrm{C}^{5+}$ at the laboratory wavelengths of $18.97 \AA$ and $33.74 \AA$, respectively. In Fig. 5, we show the spectrum around these lines, and the best-fit model. The broad troughs of these lines from $\sim 0$ to $-2200 \mathrm{~km} \mathrm{~s}^{-1}$ nicely demonstrate the velocity distribution of the outflow of NGC 7469, as well as the intricate overlap between intrinsic absorption and emission lines. The $-2050 \mathrm{~km} \mathrm{~s}^{-1}$ component is marginally 

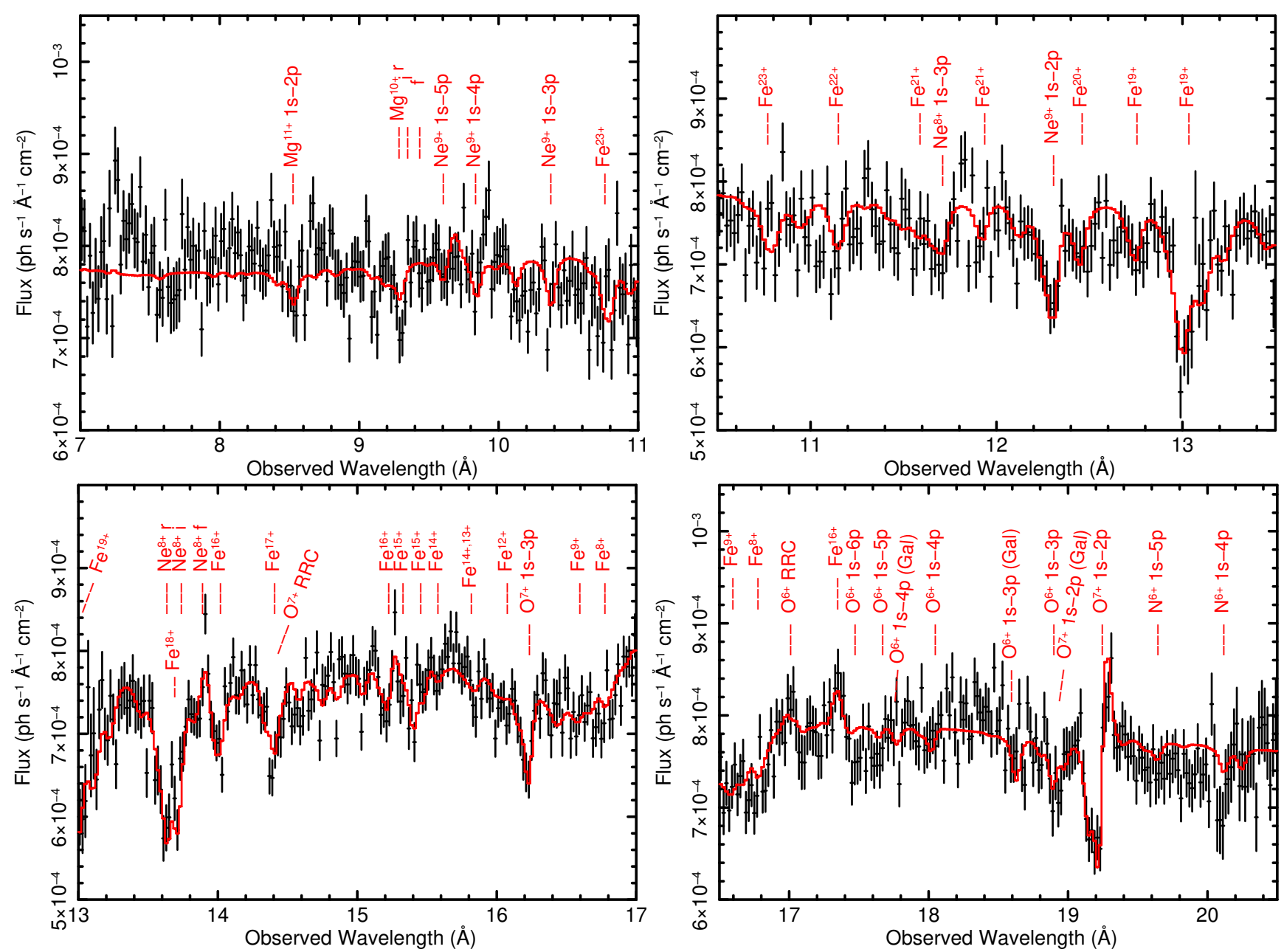

Fig. 3. Segments of the RGS spectrum of NGC 7469 with the best-fit folded model overlaid. Spectra are presented in the observed (redshifted) frame. Prominent features are marked on the spectrum at their positions in the rest frame of NGC 7469. We note the varying vertical scale from one panel to the next, none of which reach zero. Longer wavelengths are presented in Fig. 4.

resolved for $\mathrm{C}^{5+}$ thanks to the superior resolving power of the gratings at longer wavelengths $\left(\sim 600 \mathrm{~km} \mathrm{~s}^{-1}\right.$ vs. $\sim 1000 \mathrm{~km} \mathrm{~s}^{-1}$ for $\mathrm{O}^{7+}$ ).

The model also includes the photo-ionized emission component that is discussed in more detail below (Sect. 4.4). Interestingly, the emission lines observed in NGC 7469 do not seem to be at rest with respect to the host galaxy, but are blue-shifted by $-450 \mathrm{~km} \mathrm{~s}^{-1}$. Unlike the narrow absorption profiles, the emission lines are moderately broad and parametrized in the model with a turbulent velocity of $v_{\text {turb }}=600 \mathrm{~km} \mathrm{~s}^{-1}$, which corresponds to full width at half maximum (FWHM) of $\approx 1400 \mathrm{~km} \mathrm{~s}^{-1}$.

Due to instrumental broadening, there is blending of the $-650 \mathrm{~km} \mathrm{~s}^{-1}$ and $-950 \mathrm{~km} \mathrm{~s}^{-1}$ absorption troughs, as well as with the emission at $-450 \mathrm{~km} \mathrm{~s}^{-1}$. This makes the fitted parameters of these components in the model somewhat degenerate. Blustin et al. (2007) did not identify a $-950 \mathrm{~km} \mathrm{~s}^{-1}$ component, and in the present spectrum as well, it could be part of the $-650 \mathrm{~km} \mathrm{~s}^{-1}$ component (or some in-between velocity); but see attempts to tie the two below. We tend to believe these velocity components since they are consistent with the UV ones, and since they are obtained from a rather constraining global fit to the entire X-ray spectrum, and not just from these individual lines.

\subsection{Ionization and column density}

The parameters of the six absorption components, which are associated here with three different outflow velocities, are listed in
Table 2. The statistical significance of each component is given in the last column, in terms of the increase of the C-statistic $(\Delta C)$ when that component is omitted from the model. The slowest $-650 \mathrm{~km} \mathrm{~s}^{-1}$ velocity component has a broad ionization distribution of $-0.6 \lesssim \log \xi \lesssim 2.0$ represented here by three ionization components, with the column density slowly increasing with ionization, and with a possible gap in thermally unstable $\xi$ values. A similar trend was observed in the 2004 observation (Blustin et al. 2007), as well as in other Seyfert outflows (Holczer at al. 2007; Behar 2009; Fukumura et al. 2010; Laha et al. 2014). An elaborate analysis of the absorption measure distribution (AMD) is deferred to a following paper that will use a more sensitive ion-by-ion fit.

The highest ionization $(\log \xi=2.7)$ and highest column density $\left(2.2 \times 10^{21} \mathrm{~cm}^{-2}\right)$ is found in the $-950 \mathrm{~km} \mathrm{~s}^{-1}$ component. Its most notable feature is the $\mathrm{Fe}^{19+}$ blend at $12.85 \AA$, observed at $13.05 \AA$ (Fig. 3), which is one of the strongest absorption features in the spectrum. This component also accounts for the higher charge states of $\mathrm{Fe}$ in the model, and contributes to the central part of the Ly $\alpha \mathrm{O}^{7+}$ trough, although the RGS can not truly resolve it from the $-650 \mathrm{~km} \mathrm{~s}^{-1}$ component (see Fig. 5). The counterpart $-857 \mathrm{~km} \mathrm{~s}^{-1}$ component in the UV is observed only in Ly $\alpha$, which also indicates high ionization, lending support to the connection between these two X-ray and UV components. The high-ionization at $-950 \mathrm{~km} \mathrm{~s}^{-1}$ may also be, in fact, the extension of the broad ionization distribution of the $-650 \mathrm{~km} \mathrm{~s}^{-1}$ 

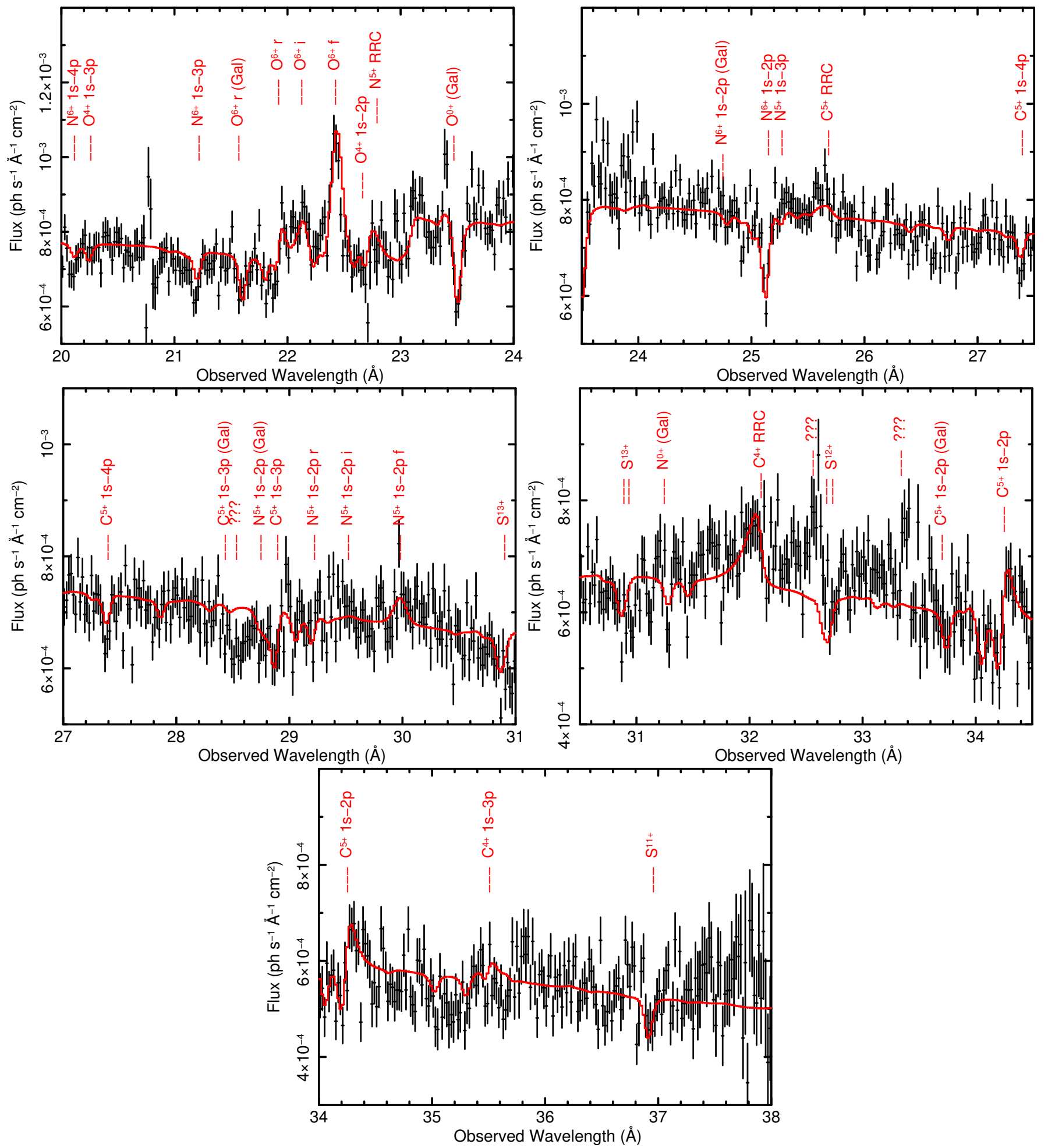

Fig. 4. Same as Fig. 3, but for wavelengths longer than $20 \AA$.

component, as its column density continues to increase with $\xi$. When forcing all four ionization components to share the same outflow velocity, the model for the entire spectrum converges to a velocity of $-830 \mathrm{~km} \mathrm{~s}^{-1}$, since much of the column is in the $-950 \mathrm{~km} \mathrm{~s}^{-1}$ component, but the fit worsens by $\Delta C \approx 30$.

The fastest component $\left(-2050 \mathrm{~km} \mathrm{~s}^{-1}\right)$ has one main ionization component with $\log \xi=2.0 \pm 0.3$, and a low column density of $\sim 10^{20} \mathrm{~cm}^{-2}$. A second, marginally significant lowionization component can be added at this velocity. It can be seen, for example, to capture the blue side of the trough of the $\mathrm{O}^{6+}$ resonance line. It improves the fit by $\Delta C \approx 50$. The fitted model in Blustin et al. (2007) indicated relatively low ionization $\left(\log \xi=0.8_{-0.3}^{+0.4}\right)$ for this fast component. However, we identify it clearly in the H-like Ly $\alpha$ lines as seen in Fig. 5. A more careful analysis of changes in the absorption troughs between 2004 and 2015 , to be reported in a separate paper, is required before determining whether or not the ionization has changed between the two epochs. The total column density in all of the present 

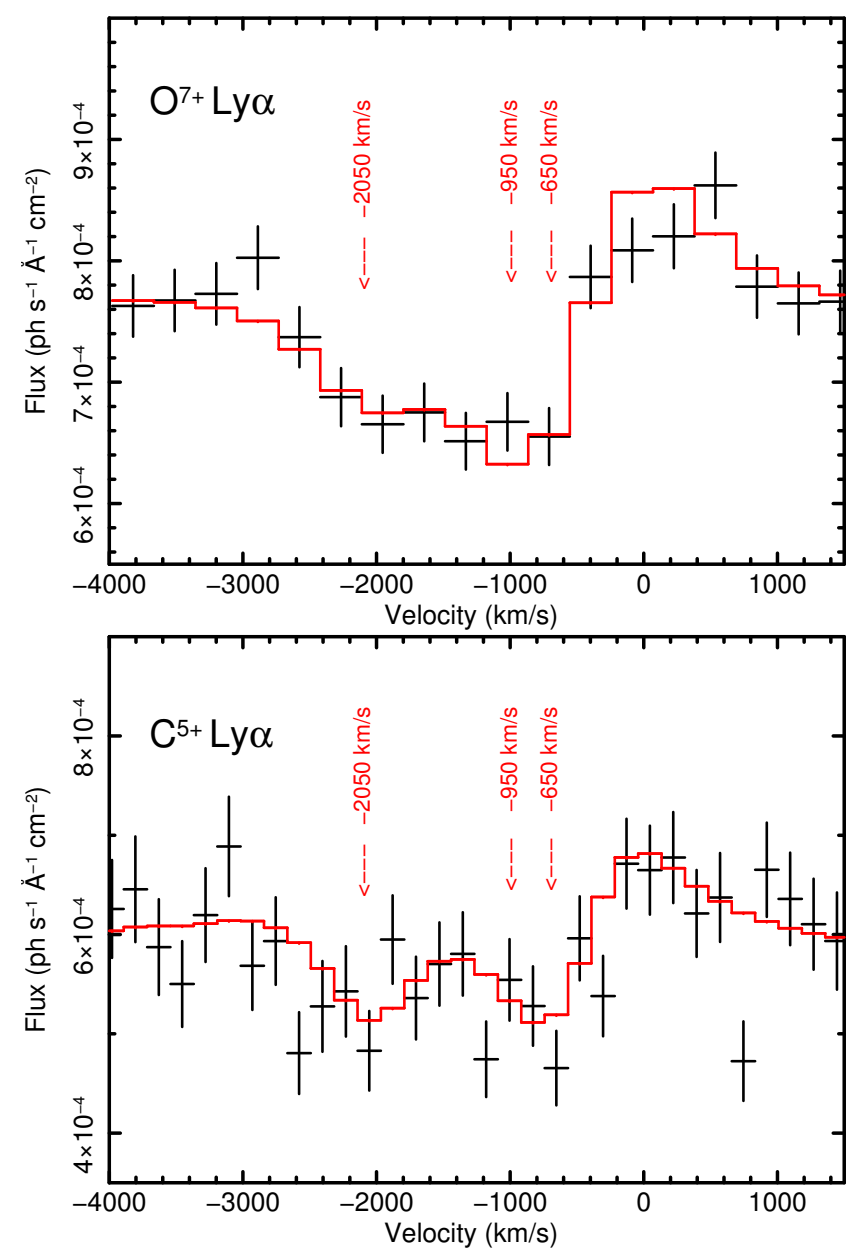

Fig. 5. Spectral region of the H-like $\mathrm{O}^{7+}$ and $\mathrm{C}^{5+} \mathrm{Ly} \alpha$ lines demonstrating the contribution of the three absorption components (marked in figure) and a broad ( $F W H M=1400 \mathrm{~km} \mathrm{~s}^{-1}$ ) emission component (not marked) centered at $-450 \mathrm{~km} \mathrm{~s}^{-1}$. The instrumental spectral resolution is $\sim 1000 \mathrm{~km} \mathrm{~s}^{-1}$ at the $\mathrm{O}^{7+}$ line and $\sim 600 \mathrm{~km} \mathrm{~s}^{-1}$ at the $\mathrm{C}^{5+}$ line, as can be seen from the folded model (solid line). Note that the vertical flux scale does not go down to zero.

components adds up to $\sim 3 \times 10^{21} \mathrm{~cm}^{-2}$, which is similar to the result of the AMD analysis of the 2004 spectrum in Blustin et al. (2007).

\subsection{Elemental abundances}

We fixed the abundance of $\mathrm{O}$ to its solar value and fitted for the abundances of $\mathrm{C}, \mathrm{N}, \mathrm{Ne}, \mathrm{Mg}, \mathrm{S}$, and $\mathrm{Fe}$, all of which have significant features in the RGS spectrum. Other elements were left at their solar values. For the sake of simplifying the model, we also tied the abundances of the emission components to those of the absorber. The abundances we obtained are $1.6 \pm 0.4,1.6 \pm 0.6$, $1.8 \pm 0.5,0.3 \pm 0.2,0.8 \pm 0.3$, and $1.4 \pm 0.4$, for $\mathrm{C}, \mathrm{N}, \mathrm{Ne}, \mathrm{Mg}$, $\mathrm{S}$, and Fe, respectively. All abundances are given with respect to the solar values of Asplund et al. (2009), and in effect represent $A_{\mathrm{Z}} / A_{\mathrm{O}}$ abundance ratios (and not absolute abundances $A_{\mathrm{Z}} / A_{\mathrm{H}}$ ). If the abundance ratio $A_{\mathrm{O}} / A_{\mathrm{H}}$ is solar, the results seem to have a slight tendency for super-solar abundances, except for $\mathrm{Mg}$ and $\mathrm{S}$. Note that the $\mathrm{Mg}$ lines around $9 \AA$ and the $\mathrm{S}$ lines of $\mathrm{S}^{13+, 12+, 11+}$ above $30 \AA$, are in regions where the RGS is less sensitive.

\subsection{Emission features}

There are several conspicuous emission lines in the spectrum, most notably the H-like Ly $\alpha$ lines of $\mathrm{O}^{7+}$ and $\mathrm{C}^{5+}$ (observed, respectively, at $\sim 19.3$ and $34.3 \AA$ ) and the so-called He-like triplet of $\mathrm{O}^{6+}$ and $\mathrm{Ne}^{8+}$, the strongest of which are the forbidden lines, indicative of recombination line driving, and photoionization conditions. All three $\mathrm{O}^{6+}$ lines are apparent in the spectrum around $22 \AA$, while only the forbidden line of $\mathrm{Ne}^{8+}$ around $13.9 \AA$ can be discerned due to blending with Fe absorption lines in that region. Bright narrow radiative recombination continua $(\mathrm{RRCs})$ at $32.1 \AA\left(\mathrm{C}^{4+}\right), 25.7 \AA\left(\mathrm{C}^{5+}\right), 22.8 \AA$ $\left(\mathrm{N}^{5+}\right), 17.0 \AA\left(\mathrm{O}^{6+}\right)$, and $14.4 \AA\left(\mathrm{O}^{7+}\right)$ can also be identified in the spectrum. All wavelengths are quoted in the observed, redshifted frame of Figs. 3 and 4.

We are able to model all of these emission features with two ionization (photemis) components, whose details are listed in Table 3 (and which also includes the hot plasma component of the following section). The lines require non-thermal broadening, which is parametrized in the model with a turbulent velocity of $v_{\text {turb }}=600 \mathrm{~km} \mathrm{~s}^{-1}$ that probably represents a distribution of projections from the ionization cone, rather than actual turbulence. The ionization parameters of the two emission components are $\log \xi=-1$ and $1 \mathrm{erg} \mathrm{s}^{-1} \mathrm{~cm}$, each contributing to the spectrum as can be seen by their statistical significance $\Delta C$ (when omitted) in the last column of Table 3. This likely represents a range of $\xi$ values. It is reminiscent of the range of the absorption components, but excluding the highest Fe-L charge states, whose line emission is less efficient in recombination (see Sect. 4.5). The abundances of these emission components, therefore, were fixed to those of the absorber, assuming they originate from the same body of gas.

\subsection{Emission from star formation}

After fitting two emission components from photo-ionized gas, we are still left with weak yet significant residuals in the $\mathrm{Fe}^{16+}$ line at $15.25 \AA$ and the $17.38 \AA$ doublet (both observed frame), which are shown in Fig. 6. The L-shell lines in photo-ionized plasmas, where they form by means of recombination, are weak relative to K-shell lines (Kallman et al. 1996; Kinkhabwala et al. 2002). The lower cosmic abundance of Fe compared to $\mathrm{C}, \mathrm{N}$, and $\mathrm{O}$ also increases this effect, although in collisionally-ionized plasmas, the Fe-L resonance lines have high collision strengths that compensate for the lower abundance, and are therefore prominent in astrophysical spectra (e.g., Behar et al. 2001). We therefore interpret these lines as arising from the star-formation ring in the center of NGC 7469 (Díaz-Santos et al. 2007).

Adding a collisionally-ionized component (APEC, Smith et al. 2001) provides a good fit to these lines. Fig. 6 can be compared with that spectral region in Fig. 3, where the model includes the collisional component. The turbulent velocity broadening can not be constrained and is fixed at $v_{\text {turb }}=100 \mathrm{~km} \mathrm{~s}^{-1}$. As in the photo-ionized components, this broadening may represent a kinematic spread of velocities projected along the line of sight, rather than actual turbulence. The best-fit temperature corresponds to $0.35 \pm 0.03 \mathrm{keV}$. The Fe-L lines are slightly blueshifted by $-250 \pm 120 \mathrm{~km} \mathrm{~s}^{-1}$. Although only marginally indicative of an outflow, this component can be associated with a starburst wind. This component also produces some flux in $\mathrm{O}^{7+}$ that blends with the emission by the photo-ionized gas. Since only the $\mathrm{Fe}^{16+}$ lines are unambiguously ascribed to the collisional gas, we are not able to fit for its abundances, and not even 
Table 2. Outflow absorption components in NGC 7469.

\begin{tabular}{cccccr}
\hline \hline $\begin{array}{c}\text { Comp. } \\
\#\end{array}$ & $\begin{array}{c}v_{\text {out }}{ }^{a} \\
\left(\mathrm{~km} \mathrm{~s}^{-1}\right)\end{array}$ & $\begin{array}{c}v_{\text {turb }} \\
\left(\mathrm{km} \mathrm{s}^{-1}\right)\end{array}$ & $\begin{array}{c}\log \xi \\
\left(\mathrm{erg} \mathrm{s}^{-1} \mathrm{~cm}\right)\end{array}$ & $\begin{array}{c}N_{\mathrm{H}} \\
\left(10^{20} \mathrm{~cm}^{-2}\right)\end{array}$ & $\Delta C$ \\
\hline 1 & $-650 \pm 50$ & $70 \pm 10$ & $-0.6 \pm 0.2$ & $0.2 \pm 0.1$ & 33 \\
2 & $\ldots$ & $70 \pm 10$ & $1.4 \pm 0.1$ & $1.0 \pm 0.3$ & 221 \\
3 & $\ldots$ & $70 \pm 10$ & $2.0 \pm 0.1$ & $5.5 \pm 1.0$ & 1027 \\
4 & $-950_{-100}^{+50}$ & $35 \pm 20$ & $2.7 \pm 0.2$ & $22 \pm 10$ & 383 \\
5 & $-2050_{-160}^{+50}$ & $60 \pm 30$ & $2.0 \pm 0.3$ & $1.1 \pm 0.3$ & 82 \\
6 & $\ldots$ & $60 \pm 30$ & $0.3 \pm 0.2$ & $0.1 \pm 0.1$ & 48 \\
\hline
\end{tabular}

Notes. ${ }^{(a)}$ Velocities and widths of components 1-3 and those of 5-6 are tied.

Table 3. Model emission components.

\begin{tabular}{ccccc}
\hline \hline $\begin{array}{c}\text { Model } \\
\text { component }\end{array}$ & $\begin{array}{c}v_{\text {out }}{ }^{a} \\
\left(\mathrm{~km} \mathrm{~s}^{-1}\right)\end{array}$ & $\begin{array}{c}\log \xi \\
\left(\mathrm{erg} \mathrm{s}^{-1} \mathrm{~cm}\right)\end{array}$ & $\begin{array}{c}E M \\
\left(10^{63} \mathrm{~cm}^{-3}\right)\end{array}$ & $\Delta C$ \\
\hline photemis & $-470 \pm 150$ & $-1.0 \pm 0.2$ & 111 \\
photemis & $\cdots$ & $1.0 \pm 0.2$ & 270 \\
\hline \multicolumn{5}{c}{$k T(\mathrm{keV})$} \\
\hline APEC & $-250 \pm 120$ & $0.35 \pm 0.03$ & $4.0 \pm 1.5$ & 65 \\
\hline
\end{tabular}

Notes. ${ }^{(a)}$ photemis velocities tied.

for its overall metallicity. We therefore tie its metallicity to the $\mathrm{Fe}$ abundance of the photo-ionized components, which is fitted to be $1.4 \pm 0.4$ with respect to $\mathrm{O}$, and in solar units (see Sect. 4.3).

The X-ray flux implied by the collisionally-ionized plasma model in the RGS band is $1.4 \times 10^{-13} \mathrm{erg} \mathrm{s}^{-1} \mathrm{~cm}^{-2}$, which corresponds to a luminosity of $L_{\mathrm{X}}=7 \times 10^{40} \mathrm{erg} \mathrm{s}^{-1}$. Star-formation $\mathrm{X}$-ray luminosity tends to correlate with the Far IR luminosity $L_{\mathrm{FIR}} \sim 10^{3.7} L_{\mathrm{X}}$ (David et al. 1992; Ranalli et al. 2003). In NGC 7469, $L_{\text {FIR }}=7 \times 10^{44} \mathrm{erg} \mathrm{s}^{-1}$ (David et al. 1992, corrected for distance), so that $L_{\mathrm{FIR}} / L_{\mathrm{X}}=10^{4}$, which is just between the two archetypical, nearby starburst galaxies M 82 and NGC 253, for which $L_{\mathrm{FIR}} / L_{\mathrm{X}}=0.4 \times 10^{4}$ and $1.8 \times 10^{4}$, respectively (David et al. 1992). Thus, the X-rays emitted by the star formation in NGC 7469 are dominated by the AGN $\left(L_{X}=\right.$ $2 \times 10^{43} \mathrm{erg} \mathrm{s}^{-1}$ ), but are exactly as expected from the FIR starformation emission.

Using an angular distance of $63.2 \mathrm{Mpc}$, the collisional component yields an emission measure $\left(E M=n_{\mathrm{e}} n_{\mathrm{H}} V\right.$, density square times volume) of $4 \times 10^{63} \mathrm{~cm}^{-3}$. This is more than ten times lower than that of the photo-ionized gas (see Sect. 4.8 below), but much higher than $7.5 \times 10^{61} \mathrm{~cm}^{-3}$, that was measured for NGC 253 (Pietsch et al. 2001). This result is consistent with the similarly higher FIR and X-ray luminosities in NGC 7469, and manifests its higher star-formation rate than M 82 and NGC 253. For a typical interstellar density of $1 \mathrm{~cm}^{-3}$, the above $E M$ corresponds to a volume of approximately $1 \mathrm{kpc}^{3}$, which nicely matches the $1 \mathrm{kpc}$ diameter of the circum-nuclear star forming ring in NGC 7469 (Díaz-Santos et al. 2007).

\subsection{Galactic absorption}

For the neutral absorption through our galaxy, we use a column density of $5.5 \times 10^{20} \mathrm{~cm}^{-2}$ that includes both $\mathrm{HI}$ and $\mathrm{H}_{2}$ (see end of Sect. 1). We use the tbabs model (Wilms et al. 2000) in Xspec with solar abundances, as its position of the neutral O K-shell

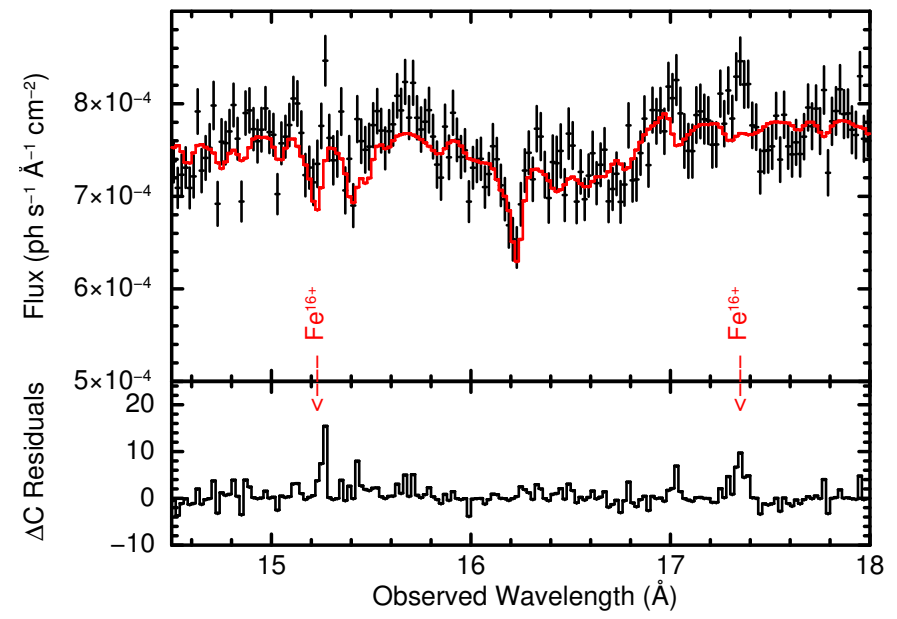

Fig. 6. Spectrum of NGC 7469 with a model that does not include the collisionally-ionized plasma component. Residuals of the $\mathrm{Ne}$-like $\mathrm{Fe}^{16+}$ $2 \mathrm{p}-3 \mathrm{~d}$ line at 15.01 and $2 \mathrm{p}-3 \mathrm{~s}$ line at $17.1 \AA$ (cosmologically redshifted in the figure) demonstrate the presence of collisionally-ionized plasma, likely originating in the circum-nuclear star-forming ring of NGC 7469.

edge at approximately $23.1 \AA$ better describes the spectrum of NGC 7469 than the $23.5 \AA$ edge in other models (see Fig. 4). The newer model tbnew brings the edge to slightly shorter wavelengths due to absorption lines, but does not improve the fit. Since tbabs does not include absorption lines, we added the $\mathrm{K} \alpha$ absorption lines of neutral $\mathrm{O}$ and $\mathrm{N}$ to the model at $23.5 \AA$ and $31.3 \AA$, respectively. These are prominent in the spectrum (Fig. 4), and do not suffer from blending with the AGN absorber (as opposed to Mrk 509, Kaastra et al. 2011; Pinto et al. 2012). The exact same method was used successfully by Reeves et al. (2016) for the X-ray spectrum of Ark 120.

It is puzzling that the fit prefers $(\Delta C=350)$ a much lower column of $3.4 \times 10^{20} \mathrm{~cm}^{-2}$ than the nominal $5.5 \times 10^{20} \mathrm{~cm}^{-2}$. The improvement is especially seen at longer wavelengths (>20 ^), and is likely driven by the $\mathrm{N}$ and $\mathrm{C}$ edges. This could indicate non-solar ISM abundances, calibration issues, or an inappropriate continuum model. Attempts to add a soft excess and then to absorb it did not improve the global fit. We fitted the archival 2004 RGS spectrum of NGC 7469 to find that, unlike here, the higher neutral column is obtained as expected. We defer a more detailed study of the local, neutral absorption to a combined X-ray and UV analysis, as was done for Mrk 509 (Pinto et al. 2012).

We identify a strong He-like $\mathrm{O}^{6+}$ absorption line in the spectrum at $21.6 \AA$, which is ascribed to hot gas in the Galactic 
halo, or in the local group. The $\mathrm{H}$-like $\mathrm{O}^{7+}$ Ly $\alpha$ line at $18.97 \AA$ is marginally present, immediately next to the (redshifted) $\mathrm{O}^{6+}$ $\mathrm{He} \beta$ line of the NGC 7469 outflow. We thus add a collisionallyionized XSTAR absorption component (hotabs) to represent this local $(z=0)$ absorber. The improvement to the global fit is $\Delta C=65$ for over 1500 degrees of freedom. The fitted parameters of this component are a temperature of $1.5 \times 10^{6} \mathrm{~K}$ and a $\mathrm{H}$ column density of $2 \times 10^{19} \mathrm{~cm}^{-2}$, under the assumption of solar abundances, which adequately accounts for the weak observed features.

\subsection{Unidentified lines}

The RGS spectrum has a bonafide absorption line at $28.56 \pm$ $0.02 \AA$ (see Fig. 4), which corresponds to $\sim 28.10 \AA$ in the rest frame of NGC 7469. There is no obvious identification at these wavelengths. The nearby $\mathrm{C}^{5+}$ Ly $\beta$ line at $28.466 \AA$ would need to be approaching at $-3800 \mathrm{~km} \mathrm{~s}^{-1}$ in NGC 7469 , or receding by $1000 \mathrm{~km} \mathrm{~s}^{-1}$ in our frame, to produce the observed feature; neither velocity is observed in other lines, including in $\mathrm{C}^{5+}$ Ly $\alpha$. A closer line is the $2 \mathrm{p}-4 \mathrm{~d}$ line of $\mathrm{Ar}^{10+}$ at $28.529 \AA$, but this ion would also have a stronger $2 \mathrm{p}-3 \mathrm{~d}$ line at $34.328 \AA$ (Lepson et al. 2003), which is not observed. There is no prominent line that we know of close to $28.10 \AA$.

The RGS spectrum also features two bright emission lines observed around $32.6 \AA$ and $33.4 \AA$, which can be seen rising high above the best fit model in Fig. 4. To measure their positions and fluxes, we fitted the lines with two Gaussians. The line widths indicate that they are unresolved. The best fitted positions are $32.60 \pm 0.02 \AA$ and $33.37 \pm 0.02 \AA$, or $32.08 \pm 0.02 \AA$ and $32.84 \pm 0.02 \AA$ in the rest frame of NGC 7469. The fluxes (corrected for Galactic absorption) are $(4.3 \pm 1.0) \times 10^{-5}$ and $(2.4 \pm 0.7) \times 10^{-5} \mathrm{erg} \mathrm{s}^{-1} \mathrm{~cm}^{-2}$, respectively.

We struggle to provide convincing interpretations for these emission lines. The only bright lines in this region of the spectrum are the K-shell lines of $\mathrm{C}$, and in particular the $\mathrm{He} \gamma$ and He $\delta$ lines of $\mathrm{C}^{4+}$ at $32.754 \AA$ and $33.426 \AA$. If ascribed to $\mathrm{C}^{4+}$, the observed lines are blue-shifted by $\sim 160$ and $50 \mathrm{m \AA}$, but narrow emission lines in the gratings can only arise from a point source along the line of sight towards NGC 7469, so this is ruled out. We could not identify possible emission lines that would occur at $32.08 \AA$ and $32.84 \AA$ in the rest frame of NGC 7469 .

\subsection{Location and physical state of the outflow}

One of the main goals of the present campaign is to track down the position of the outflow in order to determine its mass outflow rate and kinetic power, and thereby its possible influence on the host galaxy. A major attempt will be made in a separate paper discussing the variability of the absorber, which is subtle. Here, we limit ourselves to simple considerations. Most generally, the column density $N_{\mathrm{H}}$ as deduced from a single ion that forms at an ionization parameter $\xi$, can be written as

$N_{\mathrm{H}}=\int_{r_{\text {min }}}^{r_{\max }} n_{\mathrm{H}} \mathrm{d} r=\frac{L}{\xi}\left(\frac{1}{r_{\min }}-\frac{1}{r_{\max }}\right)$,

If $r_{\text {max }} \gg r_{\text {min }}$ then

$r_{\min } \approx\left(\frac{1}{r_{\min }}-\frac{1}{r_{\max }}\right)^{-1}=\frac{L}{\xi N_{\mathrm{H}}}$.

Given an ionizing X-ray luminosity of $2 \times 10^{43} \mathrm{erg} \mathrm{s}^{-1}$ and a total column density of $3 \times 10^{21} \mathrm{~cm}^{-2}$, Eq. (2) would put the present highest ionization component of $\xi=10^{3}\left(r_{\min } \approx\right)$ a few pc away from the central source, while the lower ionization components, where most of the mass is, are as far as $10 \mathrm{kpc}$ away. This is in line with the present 2015 absorption spectrum being essentially unchanged from that of 2004 (Blustin et al. 2007).

Since we observe a wide range of $\xi$ values, clearly Eq. (2) can not tell the whole story of the outflow. Whewell et al. (2015) recently used this estimate for NGC5548 and found $r_{\min }=$ $13.9 \pm 0.6 \mathrm{pc}$. On the other hand, an estimate based on changes in UV absorption troughs in NGC 5548, assuming they are only due to varying ionizing flux over 16 years, yields a distance of $3.5 \pm 1.0 \mathrm{pc}$ for one component, 5-70 pc for three other components and two more beyond $100 \mathrm{pc}$ (Arav et al. 2015). This suggests that $r_{\max } \gg r_{\min }$ may not apply, and thus, the above estimate for NGC 7469 may also be too high.

Another way to obtain an idea of the location and density of the absorber is to associate the narrow line emitting gas with the outflow. This notion is corroborated here by the actual blueshift of the emission lines, and by the overlap of $\xi$ values. An emission-line photon flux $F_{j i}$ can be related to the volume integrated emission measure:

$E M=\int_{V} n_{\mathrm{e}} n_{\mathrm{H}} \mathrm{d} V=\frac{4 \pi d^{2} F_{j i}}{f_{q+1} A_{Z} P_{j i}}$,

where $d$ is the distance to the source. The fraction of ions in the charge state of the line $q$ is denoted by $f_{q}$ (a function of $\xi$ typically peaks at $\sim 0.5)$. $A_{Z}$ is the elemental abundance with respect to $\mathrm{H}$, and $P_{j i}$ is the line power (in units of $\mathrm{cm}^{3} \mathrm{~s}^{-1}$ ). The $E M$ of the brightest line in the spectrum of NGC 7469, namely that of the forbidden line of $\mathrm{O}^{6+}$, is $\sim 10^{65} \mathrm{~cm}^{-3}$. In the approximation of $r_{\max } \gg r_{\min }$, the $E M$ can be used to estimate the opening angle of the ionization cone (see Eq. (4) in Behar et al. 2003), but here this estimate yields an unphysical value that is above $4 \pi$, giving yet another indication that this approximation is not valid here.

On the other hand, if the absorber size $\Delta r$ is much smaller than its distance from the source $r$, that is, a cloudlet, and assuming $n_{\mathrm{H}} \approx n_{\mathrm{e}}=n$, one can approximate $E M \approx n^{2} \Delta r^{3}$ and $N_{\mathrm{H}} \approx n \Delta r$, which can be directly solved to yield

$n=N_{\mathrm{H}}^{3} / E M$.

Using the above values for $\mathrm{O}^{6+}$, Eq. (4) yields $n=0.3 \mathrm{~cm}^{-3}$. Since $\mathrm{O}^{6+}$ forms at approximately $\xi=30$, this implies a distance from the center of $r=300 \mathrm{pc}$; a result to be taken with caution, as a uniform cloudlet is clearly over simplistic. For one, a strong density gradient would need to be present inside to explain the large range of $\xi$ observed (see, e.g., Różańska et al. 2006; Stern et al. 2014).

\section{Discussion and conclusions}

We show that the high-resolution X-ray spectrum of NGC 7469 requires a multitude of components that include beyond neutral and ionized absorption at $z=0$, several components of ionized absorption in the outflow of the AGN, low and high photo-ionization emission components, and X-ray lines from the circumnuclear starburst of NGC 7469, and its wind. The main results of this analysis can be summarized as follows:

- The outflow velocities of $-650,-950$, and $-2050 \mathrm{~km} \mathrm{~s}^{-1}$ are consistent with the three components detected simultaneously in the UV, although the presence of the $-950 \mathrm{~km} \mathrm{~s}^{-1}$ component is not unambiguous in the X-ray spectrum, and could also be associated with an extension to high ionization of the $-650 \mathrm{~km} \mathrm{~s}^{-1}$ component. 
- The absorber of NGC 7469 shows a broad ionization distribution, at least in the slowest velocity component. This is evidently a ubiquitous feature of Seyfert outflows.

- Preliminary estimates of the position of the absorber indicate it is a few pc away from the center or more, but a careful variability study in the X-rays and the UV will provide better constraints.

- The narrow emission lines from photo-ionized gas are blueshifted, indicating that they may originate from the absorbing outflow. This X-ray narrow line region is likely the same extended region observed in the UV and resolved in nearby Seyfert galaxies (e.g., Ruiz et al. 2005).

- Subtle but significant emission lines are detected from the hot circumnuclear starburst ring of NGC 7469. The high flux and emission measure of this plasma indicates star formation that exceeds nearby starbursts.

Future work will seek and analyze small changes in absorption over the course of the months-long 2015 campaign, and make comparisons with the 2004 observation. X-ray absorption variability will be compared with a similar analysis of the UV HST/COS spectrum of NGC 7469, where the S/N is much better. The goal is to identify a change in the X-ray and UV absorbers that can be ascribed to changes in the ionizing continuum, and thus to obtain an idea of the density, distance from the center, and, in turn, the mass outflow rate.

Acknowledgements. This work is supported by NASA grant NNX16AC07G through the XMM-Newton Guest Observing Program, and through grants for HST program number 14054 from the Space Telescope Science Institute, which is operated by the Association of Universities for Research in Astronomy, Incorporated, under NASA contract NAS5-26555. The research at the Technion is supported by the I-CORE program of the Planning and Budgeting Committee (grant number 1937/12). N.A. is grateful for a visiting-professor fellowship at the Technion, granted by the Lady Davis Trust. E.B. received funding from the European Union's Horizon 2020 research and innovation programme under the Marie Sklodowska-Curie grant agreement No. 655324. S.B. and M.C acknowledge financial support from the Italian Space Agency under grant ASIINAF I/037/12/0. P.O.P. and F.U. acknowledge support from CNES and from PNHE of CNRS/INSU. G.P. acknowledges support by the Bundesministerium für Wirtschaft und Technologie/Deutsches Zentrum für Luft- und Raumfahrt (BMWI/DLR, FKZ 50 OR 1408 and FKZ 50 OR 1604) and the Max Planck Society. We thank the referee for useful comments that have been implemented in the manuscript.

\section{References}

Arav, N., Brotherton, M. S., Becker, R. H., et al. 2001, ApJ, 546, 140 Arav, N., Chamberlain, C., Kriss, G. A., et al. 2015, ApJ, 577, 37 Asplund, M., Grevesse, N., Sauval A. J., \& Scott P. 2009, ARA\&A, 47, 481
Behar, E. 2009, ApJ, 703, 1346

Behar, E., Cottam, J. C., \& Kahn, S. M. 2001, ApJ, 548, 966

Behar, E., Rasmussen, A. P., Blustin, A. J., et al. 2003, ApJ, 598, 232

Blustin, A. J., Page, M. J., Fuerst, S. V., Branduardi-Raymont, G., \& Ashton, C. E. 2005, A\&A, 431, 111

Blustin, A. J., Kriss, G. A., Holczer, T., et al. 2007, A\&A, 466, 107

Contopoulos, J., \& Lovelace, R. V. E. 1994, ApJ, 429, 139

Crenshaw, D. M., Kraemer, S. B., \& George, I. M. 2003, ARA\&A, 41, 117

David, L. P., Jones, C., \& Forman, W. 1992, ApJ, 388, 82

Detmers, R. G., Kaastra, J. S., Steenbrugge, K. C., et al. 2011, A\&A, 534, A38

Díaz-Santos, T., Alonso-Herrero, A., Colina, L., Ryder, S. D., \& Knapen, J. H. 2007, ApJ, 661, 149

Di Matteo, T., Springel, V., \& Hernquist, L. 2005, Nature, 433, 604

Ebrero, J., Costantini, E., Kaastra, J. S., et al. 2010, A\&A, 520, A36

Fukumura, K., Kazanas, D., Contopoulos, I., \& Behar, E. 2010, ApJ, 715, 636

Gabel, J. R., Arav, N., Kaastra, J. S., et al. 2005, ApJ, 623, 85

Holczer, T., Behar, E., \& Kaspi, S. 2007, ApJ, 663, 799

Kaastra, J. S., Mewe, R., Liedahl, D. A., Komossa, S., Brinkman, A. C. 2000, A\&A, 354, L83

Kaastra, J. S., Raassen, A. J. J., Mewe, R., et al. 2004, A\&A, 428, 57

Kaastra, J. S., Petrucci, P.-O., Cappi, M., et al. 2011, A\&A, 534, A36

Kaastra, J. S., Detmers, R. G., Mehdipour, M., et al. 2012, A\&A, 539, A117

Kaastra, J. S., Kriss, G. A., Cappi, M., et al. 2014, Science, 345, 64

Kallman, T. R., \& Bautista, M. 2001, ApJS, 133, 221

Kallman, T. R., Liedahl, D., Osterheld, A., Goldstein, W., \& Kahn, S. M. 1996, ApJ, 465, 994

Kaspi, S., Brandt, W. N., Netzer, H., et al. 2001, ApJ, 554, 216

Kinkhabwala, A., Sako, M., Behar, E., et al. 2002, ApJ, 575, 732

Krolik, J. H., \& Kriss, G. A. 2001, ApJ, 561, 684

Krongold, Y., Nicastro, F., Elvis, M., et al. 2005, ApJ, 620, 165

Krongold, Y., Nicastro, F., Elvis, M., et al. 2007, ApJ, 659, 1022

Laha, S., Guainazzi, M., Dewangan, G. C., Chakravorty S., \& Kembhavi, A. K. 2014, MNRAS, 441, 2613

Lepson, J. K., Beiresdorfer, P., Behar, E., \& Kahn, S. M. 2003, ApJ, 590, 604

McKernan, B., Yaqoob, T., \& Reynolds, C. S. 2007, ApJ, 379, 1359

Netzer, H., Kaspi, S., Behar, E., et al. 2003, ApJ, 599, 933

Pietsch, W., Roberts, T. P., Sako, M., et al. 2001, A\&A, 365, L174

Pinto, C., Kriss, G. A., Kaastra, J. S., et al. 2012, A\&A, 541, A147

Ponti, G., Papadakis, I., Bianchi, S., et al. 2012, A\&A, 542, A83

Proga, D., Stone, J. M., \& Kallman, T. R. 2000, ApJ, 543, 686

Ranalli, P., Comastri, A., \& Seti, G. 2003, A\&A, 399, 39

Reeves, J. N., O’Brien, P. T., \& Ward, M. J. 2003, ApJ, 593, L65

Reeves, J. N., Porquet, D., Braito, V., et al. 2016, ApJ, 828, 98

Różańska, A., Goosmann, R., Dumont, A.-M., \& Czerny, B. 2006, A\&A, 452, 1

Ruiz, J. R., Crenshaw, D. M., Kraemer, S. B., et al. 2005, AJ, 129, 73

Scott, J. E., Kriss, G. A. Lee, J. C., et al. 2005, ApJ, 634, 193

Sim, S. A., Long, K. S., Miller, L., \& Turner, T. J. 2008, MNRAS, 388, 611

Smith, R. K., Brickhouse, N. S., Liedahl, D. A., \& Raymond, J. C. 2001, ApJ, 556, L91

Springob, C. M., Haynes, M. P., Giovanelli, R., \& Kent, B. R. 2005, ApJS, 160, 149

Stern, J., Behar, E., Laor, A., Baskin, A., \& Holczer, T. 2014, MNRAS, 445, 3011

Tombesi, F., Cappi, M., Reeves, J. N., et al. 2010, A\&A, 521, A57

Wakker, B. P. 2006, ApJS, 163, 282

Wakker, B. P., Lockman, F. J., \& Brown, J. M. 2011, ApJ, 728, 159

Wilms, J., Allen, A., \& McCray, R. 2000, ApJ, 542, 914 